\title{
COMPUTER BASED TOOLS TO LOCATE AND MEASURE THE DISEASE INFECTED AREA OF RICE LEAVES
}

\author{
M. M. Billah, M. M. Bhuiyan and M. A. Ashraf ${ }^{1}$ \\ Department of Computer Science and Mathematics, Bangladesh Agricultural University \\ Mymensingh-2202, Bangladesh
}

\begin{abstract}
Computer based image processing system has been developed to automatically calculate the damaged area and also to identify the location of disease infected area of rice leaves. The developed image processing system separates the infected regions on leaf by pixel-wise processing. The color intensities associated with image pixels are used as inputs, which are taken by the software and produces the desired output. Experimental results show that the proposed system can produce accurate results in every case whereas conventional eye-estimation technique is not accurate. The amount of damaged area, as identified by proposed software system, can potentially be used in many decision making processes, like determination of the amount of pesticides need to be sprayed in a field. Moreover, an automated machine can be combined with this software to eliminate the requirement of human operators. Also this system may eliminate the requirement of costly Leaf Area Meter.
\end{abstract}

Key Words : Eye-estimation, Image processing, Infected leaf area, Leaf area meter

\section{INTRODUCTION}

Computer based image processing system is necessary to increase the speed of identification of damaged area and position of diseases in rice leaves. This measurement is helpful in many applications, like identifying diseases on leaves, eliminating excess use of pesticides in the rice fields and so on. The amount of pesticides needs to be sprayed in a field is determined by the percentage of damaged area of leaves (Agrios, 1997). Currently, this area is determined by human vision in Bangladesh. Though Leaf Area Meter (LAM) can be used, but its too complex and not free from human error (Meah, 2007). The computer based system in agriculture is a new approach in Bangladesh. The disease detection, status assessment, decision and control functions required by a precision pesticides application system can be performed by a human operator. But such tasks can be performed more efficiently by an automated system. A machine vision system provides an alternative to the manual inspection of biological entities. This system can be used to gather information, which can be processed, analysed, and

1 Department of Farm Structure, Bangladesh Agricultural University, Mymensingh-2202, Bangladesh 
transformed into inputs to a decisional algorithm that controls the action in real-time. Machine vision system is the technological integration of a digital camera and a computer.

Evidently, there is a need to increase the yield and improve the health quality of the crop by detecting and controlling crop disease. Among the detection practices used, computer based disease infected area detection will one of the effective, cheapest, fastest and safest methods of direct plant disease management. In recent years there has been a growing interest in computer-based image processing due to commercial applications such as face recognition, biological object recognition, person identification, access control systems, weed classifications, agricultural objects detection, etc. (Jayas et al., 2000; Yang et al., 2000; Baxes, 1994; Bernier, 2000).

This study was aimed at developing a computer-based software to calculate damaged area and the position of the disease on rice leaves without depending on human vision. It is a pioneer attempt to image processing in this field in Bangladesh. At present, these measurements are made without any tools but eye-estimation, which are not precise. Moreover, these measurements are dependent on the human performing task. But the actual decision depends on exact measurements. Therefore, a method is needed to accurately identify and measure the damaged area in leaves. In such a situation computer based tools and software system is definitely a right approach.

\section{MATERIALS AND METHODS}

A number of steps were involved to determine the damaged area in a leaf. Damaged leaves were collected from different fields. These leaves were scanned to produce their digital images in computer and saved as JPEG format. The developed software works in two steps: identification of disease infected portion, calculation of disease infected area and total leaf area. Fig. 1 shows the technique for identification of disease infected area, which paints the damaged area with black color.

Rice leaves have green color. If any portion of a healthy leaf is infected by some disease its green color fades up. By observing this faded up portion, it can be recognized as the disease infected area. When a portion of a leaf is faded up, the green color component of that region is decreased and red or blue color component is relatively increased. In digital image each pixel has red, green and blue color components. If any pixel's green component is lower than either red or blue component, it can be assumed that the pixel in question is on damaged area. So the recognized pixel is marked with black color. After inspecting all pixels, all infected regions are marked as black.

Fig. 2 shows an image of disease infected rice leaf and Fig. 3 shows the resulting image after applying above flowchart on Fig. 2. 


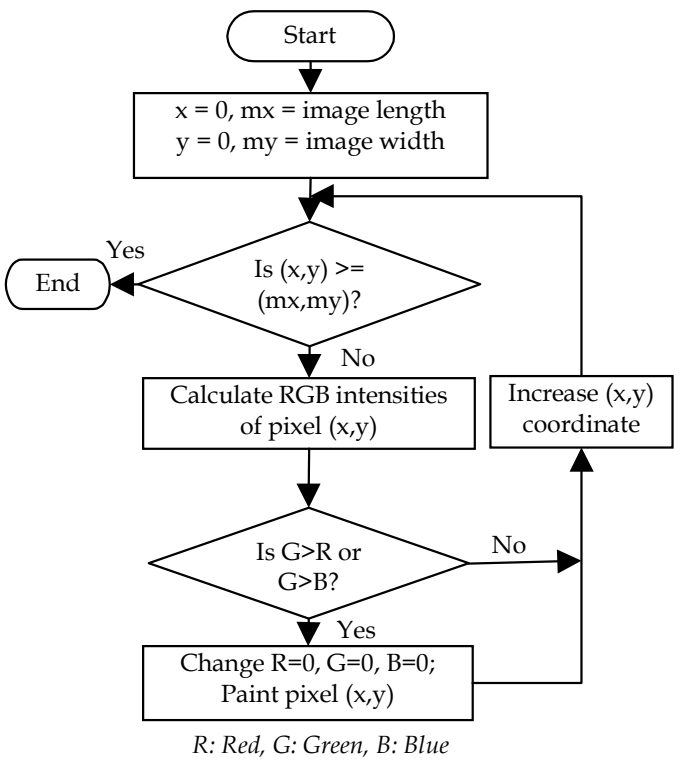

Fig. 1. Identification flowchart of disease infected area

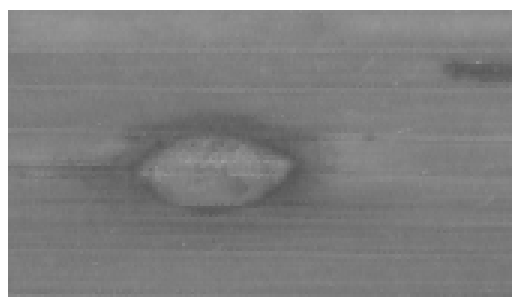

Fig. 2. Disease infected rice leaf

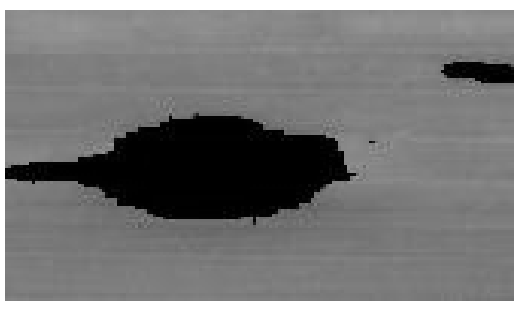

Fig. 3. After applying above flowchart over Fig. 2

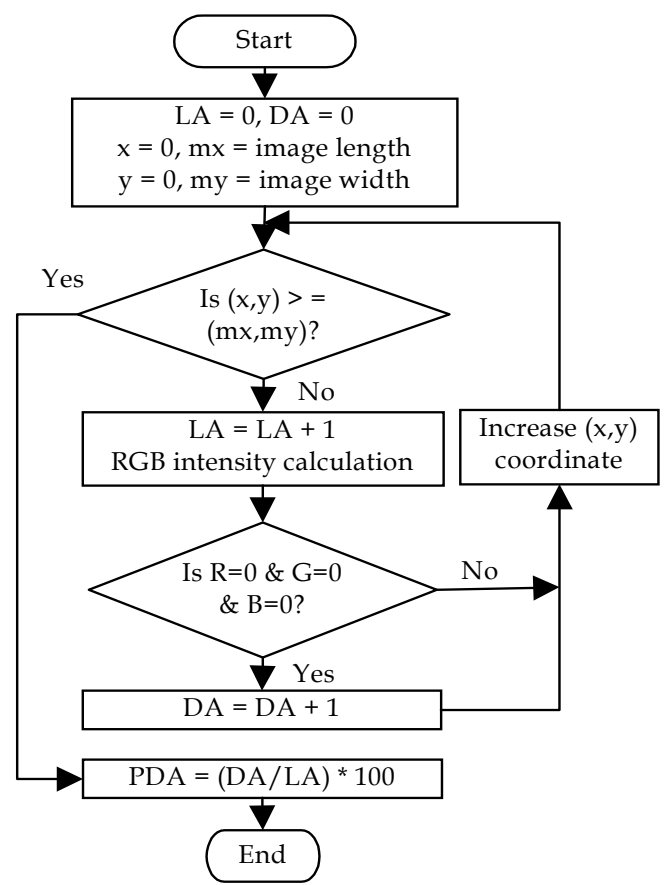

R: Red, G: Green, B: Blue

LA: Leaf area, DA: Diseased area

PDA: Percentage of Diseased area

Fig. 4. Diseased area calculation flowchart 
The next step is to calculate the percentage of total damaged area using the procedure shown in Fig. 4. It simply measures the area of black regions to calculate the percentage of damaged area. But these regions have no regular shape. Hence it is hard to accurately measure the infected area manually. However the digital image is composed a number of pixels only, which enables software to count the total no of black pixels and the total no of pixels on the leaf. Form these two measures, the percentage of damaged area is calculated.

\section{RESULTS AND DISCUSSION}

Disease infected leaves were collected from different rice fields. Rice plants of different ages and also with different disease-stages were selected randomly for the collection of leaves. The software was developed using Microsoft Visual Basic 6.0, also which was applied to identify the damaged area. Eye-estimation technique was also used for the same task using different persons. The comparative results of these two methods are shown in Table 1 and corresponding leaf images are shown in Fig. 5 (a, b and c).

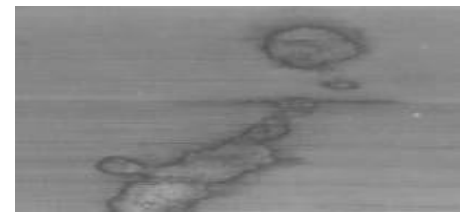

(a)

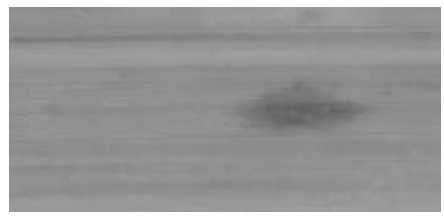

(b)

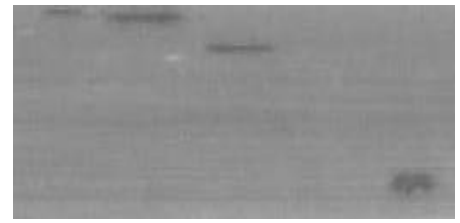

(c)

Fig. 5. Disease infected rice leaves

Table1. Comparison of proposed system to existing methods

\begin{tabular}{c|c|c|c|c}
\hline Images & $\begin{array}{c}\text { Infected area as } \\
\text { calculated by developed } \\
\text { software (\%) }\end{array}$ & $\begin{array}{c}\text { Infected area by } \\
\text { eye-estimation (\%) } \\
\text { (Person 1) }\end{array}$ & $\begin{array}{c}\text { Infected area by eye- } \\
\text { estimation (\%) } \\
\text { (Person 2) }\end{array}$ & $\begin{array}{c}\text { Infected area } \\
\text { by LAM (\%) }\end{array}$ \\
\hline Fig. 5(a) & 18.20 & 25 & 20 & 19.60 \\
Fig. 5(b) & 5.10 & 8 & 10 & 6.50 \\
Fig. 5(c) & 3.50 & 5 & 8 & 4.10 \\
\hline
\end{tabular}

Table 1 shows that eye-estimation technique is not accurate. It varies with person. This variation may be severe for some particular persons. However as the proposed system is based on computer, it produces the accurate result each time. Additional 140 samples were used and similar results to that of Table 1 were observed. 
The area measuring technique was verified for the image in Fig. 3. The developed system showed $19.20 \%$ of total damaged area. But using eye-estimation technique, it seems to be more than $30 \%$. Using LAM it was found that the area was $20.30 \%$. The LAM may be an alternative to the system. But it will slow down the total process because several iterations are needed for computation. So this method is time consuming as a single leaf has several infected regions. Moreover, the dissection process may lose some damaged area depending on human error. But the computer based system calculates area of a leaf by using one iteration without requiring any dissection.

\section{CONCLUSION}

This study was undertaken to develop a computer system to identify rice leaves disease infected area taken from the rice fields. Basic color index values were assigned to the input pixels. There were 140 samples for testing and the over all performance of the proposed system was compared with eye-estimation and LAM methods. The success rate for the measurement of disease infected area was varied $0.3 \%$ to $2.4 \%$ and $2.5 \%$ to $10.7 \%$ by LAM and eye-estimation methods respectively. Image processing systems are capable of replacing the human inspection systems because of their high speed, actual measurements, precision and indefatigable operation. Fast image processing system can be useful in the control of agricultural real-world, site-specific disease identifications, use proper amount of herbicides and related applications.

\section{REFERENCES}

Agrios, G. N. 1997. Plant Pathology : Academic Press. Boston, London.

Beranier, T. and Landry, J. A. 2000. Algorithmic recognition of biological objects. Canad. Agril. Eng., 42(2) : 101-109

Baxes, G. A. 1994. Digital Image Processing : Principles and Applications. John Wiley \& Sons Inc, New York, pp. 77-86.

Jayas, D. S., Paliwal, J. and Visen, N. S. 2000. Multi-layer Neural Networks for Image Analysis of Agricultural Products. J. Agric. Eng. Res., 77(2) : 119-128.

Meah, M. B. 2007. Personal Communication. Professor, Plant Pathology. Bangladesh Agricultural University, Mymensingh.

Yang, C. C., Prasher, S. O., Landry, J. A. and Ditommaso, A. 2000. Recognition of weeds with image processing and their use with fuzzy logic for precision farming. Canad. Agril. Eng., 42 : 195-200. 